\title{
Prognostic Value of Preoperative MRI Metrics for Diffuse Lower-Grade Glioma Molecular Subtypes
}

\author{
(D) P. Darvishi, (DP.P. Batchala, (D).T. Patrie, (DL.M. Poisson, (D) M.-B. Lopes, (D) R. Jain, (DC.E. Fadul, (DD. Schiff, and (D) S.H. Patel
}

\section{ABSTRACT}

BACKGROUND AND PURPOSE: Despite the improved prognostic relevance of the 2016 WHO molecular-based classification of lower-grade gliomas, variability in clinical outcome persists within existing molecular subtypes. Our aim was to determine prognostically significant metrics on preoperative MR imaging for lower-grade gliomas within currently defined molecular categories.

MATERIALS AND METHODS: We undertook a retrospective analysis of 306 patients with lower-grade gliomas accrued from an institutional data base and The Cancer Genome Atlas. Two neuroradiologists in consensus analyzed preoperative MRIs of each lower-grade glioma to determine the following: tumor size, tumor location, number of involved lobes, corpus callosum involvement, hydrocephalus, midline shift, eloquent cortex involvement, ependymal extension, margins, contrast enhancement, and necrosis. Adjusted hazard ratios determined the association between MR imaging metrics and overall survival per molecular subtype, after adjustment for patient age, patient sex, World Health Organization grade, and surgical resection status.

RESULTS: For isocitrate dehydrogenase (IDH) wild-type lower-grade gliomas, tumor size (hazard ratio, 3.82; 95\% Cl, 1.94-7.75; $P<.001$ ), number of involved lobes (hazard ratio, 1.70; 95\% Cl, 1.28-2.27; $P<.001$ ), hydrocephalus (hazard ratio, 4.43; $95 \% \mathrm{Cl}, 1.12-$ 17.54; $P=.034$ ), midline shift (hazard ratio, 1.16; $95 \% \mathrm{Cl}$, 1.03-1.30; $P=.013$ ), margins $(P=.031)$, and contrast enhancement (hazard ratio, 0.34; $95 \% \mathrm{Cl}, 0.13-0.90 ; P=.030$ ) were associated with overall survival. For $I D H$-mutant $1 \mathrm{p} / 19 \mathrm{q}$-codeleted lower-grade gliomas, tumor size (hazard ratio, $2.85 ; 95 \% \mathrm{Cl}, 1.06-7.70 ; P=.039$ ) and ependymal extension (hazard ratio, 6.34; $95 \% \mathrm{Cl}, 1.07-37.59 ; P=.042$ ) were associated with overall survival.

CONCLUSIONS: MR imaging metrics offers prognostic information for patients with lower-grade gliomas within molecularly defined classes, with the greatest prognostic value for IDH wild-type lower-grade gliomas.

ABBREVIATIONS: $L G G=$ lower-grade glioma; $H R=$ hazard ratio; IDH = isocitrate dehydrogenase; IDHmut-Codel $=I D H$ mutation and a whole-arm deletion of chromosome arms lp and 19q; IDHmut-Noncodel $=I D H$-mutant lacking lp/19q codeletion; IDHwt $=I D H$ wild-type; IQR $=$ interquartile range; OS overall survival; TCIA = The Cancer Imaging Archive; TERT = telomerase reverse transcriptase; WHO = World Health Organization

$\mathbf{R}$ ecognition of the biologic and prognostic significance of molecular-based characterization of diffuse lower-grade gliomas (LGGs) heralded major revisions to their classification by the World Health Organization (WHO) in 2016. ${ }^{1-3}$ LGGs encompass WHO grade II and III astrocytic and oligodendroglial tumors, most of which have a prognostically favorable mutation in the isocitrate dehydrogenase (IDH) gene. ${ }^{2,4}$ Oligodendrogliomas are defined by the presence of both an IDH mutation and a whole-

Received November 27, 2019; accepted after revision February 29, 2020

From the Departments of Radiology and Medical Imaging (P.D., P.P.B., S.H.P.); Public Health Sciences (J.T.P.); Pathology, Divisions of Neuropathology and Molecular Diagnostics (M.-B.L.); and Division of Neuro-Oncology (C.E.F., D.S.), University of Virginia Health System, Charlottesville, Virginia; Department of Public Health (L.M.P.), Henry Ford Health System, Detroit, Michigan; and Departments of Radiology (R.J.) and Neurosurgery (R.J.), New York University School of Medicine, New York, New York.

S.H. Patel was supported by the Radiological Society of North America Research Scholar Grant (RSCH1819). arm deletion of chromosome arms 1p and 19q (IDHmut-Codel), which confer added prognostic and therapeutic favorability. 5,6 $I D H$-mutant astrocytomas (IDHmut-Noncodel) lack $1 \mathrm{p} / 19 \mathrm{q}$ codeletion and typically have tumor protein p53 and alpha-thalassemia/mental retardation syndrome X-linked (ATRX chromatin remodeler, $[A T R X]$ ) gene mutations. ${ }^{7,8} I D H$ wild-type LGGs (IDHwt) are generally associated with far worse clinical outcomes and largely comprise neoplasms that are genotypically similar to primary glioblastoma. ${ }^{1,9}$

Despite improved clinical applicability of the revised classification system, there remains substantial heterogeneity in clinical outcomes within existing subtypes of LGGs. ${ }^{10-12}$ While numerous

Please address correspondence to Sohil H. Patel, MD, Department of Radiology and Medical Imaging, University of Virginia Health System, PO Box 800170 , Charlottesville, VA 22908; e-mail: shp4k@virginia.edu http://dx.doi.org/10.3174/ajnr.A6511 
studies have reported neuroimaging features that predict currently defined glioma molecular subtypes, ${ }^{13-20}$ neuroimaging features might additionally contain information that allows prognostic stratification of gliomas within currently defined molecular categories. The purpose of our investigation was to determine whether neuroimaging features on preoperative anatomic MR imaging have prognostic significance for LGGs within currently defined molecular categories.

\section{MATERIALS AND METHODS}

This was a retrospective study with institutional review board approval (University of Virginia Health System, Charlottesville, Virginia) as well as Health Insurance Portability and Accountability Act compliance.

\section{Patient Selection}

Cases were accrued from a diffuse LGG data base maintained at our institution $(n=255)$ as well as from The Cancer Imaging

Table 1: Patient characteristics for each molecular subtype

\begin{tabular}{lrrc}
\hline & IDHmut-Codel & IDHmut-Noncodel & IDHwt \\
\hline Total patients $^{\mathrm{a}}$ & $104(34.0 \%)$ & $129(42.2 \%)$ & $73(23.9 \%)$ \\
Female sex & $58(55.8 \%)$ & $61(47.3 \%)$ & $35(47.9 \%)$ \\
Age $^{\text {b }}$ & $48(17-74)$ & $35(17-70)$ & $57(19-76)$ \\
WHO grade & & & \\
II & $72(69.2 \%)$ & $83(64.3 \%)$ & $34(46.6 \%)$ \\
III & $32(30.8 \%)$ & $46(35.7 \%)$ & $39(53.3 \%)$ \\
\hline
\end{tabular}

${ }^{a}$ Data are listed as count (percentage of total cohort).

${ }^{b}$ Data are listed as median (range). Other data are listed as count (percentage of cases within given molecular subtype).

Table 2: Empiric distribution summaries for the preoperative MR imaging metrics according to LGG molecular subtype

\begin{tabular}{lccc}
\hline \multicolumn{1}{c}{ MR Imaging Metric } & $\begin{array}{c}\text { IDHmut- } \\
\text { Codel }\end{array}$ & $\begin{array}{c}\text { IDHmut- } \\
\text { Noncodel }\end{array}$ & IDHwt \\
\hline Tumor size (cm) & & & \\
$\quad$ Median & 6.6 & 6.9 & 6.2 \\
IQR (1st to 3rd quartile) & $(5.2-8.6)$ & $(5.3-9.3)$ & $(4.6-8.4)$ \\
$\quad$ Range (minimum-maximum) & $(1.9-14.2)$ & $(1.7-15.3)$ & $(1.1-15.0)$ \\
Location & & & \\
$\quad$ Central & $26(25.0 \%)$ & $43(33.3 \%)$ & $29(39.7 \%)$ \\
$\quad$ Peripheral & $78(75.0 \%)$ & $86(66.7 \%)$ & $44(60.3 \%)$ \\
No. of involved lobes & & & \\
Median & 2.0 & 2.0 & 2.0 \\
IQR (1st to 3rd quartile) & $(1.0-3.0)$ & $(1.0-3.0)$ & $(1.0-3.0)$ \\
$\quad$ Range (minimum-maximum) & $(1.0-6.0)$ & $(1.0-9.0)$ & $(1.0-8.0)$ \\
Corpus callosum extension & $44(42.3 \%)$ & $46(35.7 \%)$ & $18(24.2 \%)$ \\
Hydrocephalus & $12(11.5 \%)$ & $27(20.9 \%)$ & $7(9.6 \%)$ \\
Midline shift (cm) & & & \\
$\quad$ Median & 0.00 & 0.30 & 0.00 \\
IQR (1st to 3rd quartile) & $(0.00-0.43)$ & $(0.00-0.80)$ & $(0.00-0.10)$ \\
$\quad$ Range (minimum-maximum) & $(0.00-2.70)$ & $(0.00-1.90)$ & $(0.00-1.40)$ \\
Eloquent cortex & $33(31.7 \%)$ & $45(34.9 \%)$ & $24(32.9 \%)$ \\
Ependymal extension & $65(62.5 \%)$ & $80(62.0 \%)$ & $58(79.5 \%)$ \\
Margin & & & \\
$\quad$ <33\% sharp/circumscribed & $32(30.8 \%)$ & $31(24.0 \%)$ & $28(38.4 \%)$ \\
33\%-66\% sharp/circumscribed & $55(52.9 \%)$ & $38(29.5 \%)$ & $31(42.5 \%)$ \\
$\quad>66 \%$ sharp/circumscribed & $17(16.3 \%)$ & $60(46.5 \%)$ & $14(19.2 \%)$ \\
Contrast enhancement & $21(31.8 \%)$ & $51(39.5 \%)$ & $30(41.1 \%)$ \\
Necrosis & $17(16.3 \%)$ & $14(10.9 \%)$ & $18(24.7 \%)$ \\
\hline
\end{tabular}

Archive (TCIA) $(n=198) .{ }^{21}$ Inclusion criteria were the following: 1) known $I D H$ mutation status and $1 \mathrm{p} / 19 \mathrm{q}$-codeletion status; 2) known surgical resection status (gross total resection, subtotal resection, or biopsy); and 3) available preoperative MR imaging including (at a minimum) precontrast T1WI, contrast-enhanced T1WI, and either T2WI or FLAIR.

From the institutional dataset, 178 cases met the inclusion criteria; 43 cases were excluded for lack of IDH and/or 1p/ $19 \mathrm{q}$-codeletion status, and 34 cases were excluded for lack of requisite preoperative MR imaging. From the TCIA cohort, 128 cases met the inclusion criteria; 1 case was excluded for lack of $I D H$ and 1p/19q-codeletion status and 69 cases lacked requisite preoperative MR imaging. In total, our study cohort included 306 cases. Overall survival times were available for all included patients, defined as the time between the date of pathologic diagnosis to the date of death or last contact.

\section{Neuroimaging and Analysis}

Two neuroradiologists with 6 and 14 years of experience, blinded to molecular status, WHO grade, patient demographics, and clinical outcome, analyzed the preoperative MR imaging to determine the following metrics:-1) tumor size: maximum long-axis diameter (centimeters); 2) location: any glioma signal abnormality/enhancement involving the basal ganglia, thalamus, or brain stem (yes = central; no = peripheral); 3 ) number $(n)$ of involved lobes: each of the following counted as 1 lobe (per hemisphere)frontal lobe, parietal lobe, temporal lobe, insula, occipital lobe, brain stem/cerebellum; 4) corpus callosum involvement: any glioma signal abnormality/enhancement involving the corpus callosum (yes or no); 5) hydrocephalus: (yes or no); 6) midline shift: greatest degree of contralateral brain displacement (centimeters); 7) eloquent cortex involvement: any glioma signal abnormality/enhancement involving the eloquent cortex, defined per Chang et $\mathrm{al}^{22}$ (yes or no); 8) ependymal extension: any glioma signal abnormality/ enhancement involving the ventricular ependyma (yes or no); 9) margins: $<33 \%$, 33\%-66\%, or $>66 \%$ sharp/ circumscribed glioma margins; 10) contrast enhancement: any glioma contrast enhancement (yes or no); and 11) necrosis: any region of glioma necrosis characterized by peripheral contrast enhancement and central nonenhancement (yes or no).

\section{Neuropathology}

Pathologic data for patients in the institutional dataset were retrieved from the electronic medical record. Molecular markers were tested in the Clinical Laboratory Improvement Amendments-certified molecular pathology laboratory at our institution. A clinically validated IDH1 antibody was used to determine IDH mutation status. ${ }^{23,24}$ Clinically validated pyrosequencing assays (PyroMark Q24 system; https://www. qiagen.com/us/shop/automated-solutions/pyromark-q24/) were used in immunohistochemistrynegative cases in accordance with the manufacturers to determine IDH1/IDH2 mutation status. 
The $1 \mathrm{p} / 19 \mathrm{q}$-codeletion status was determined by fluorescence in situ hybridization; dual-color human probes localizing the 1p, 1q, $19 \mathrm{p}$, and 19q loci were used (Vysis LSI 1p36/1q25 and LSI 19q13/

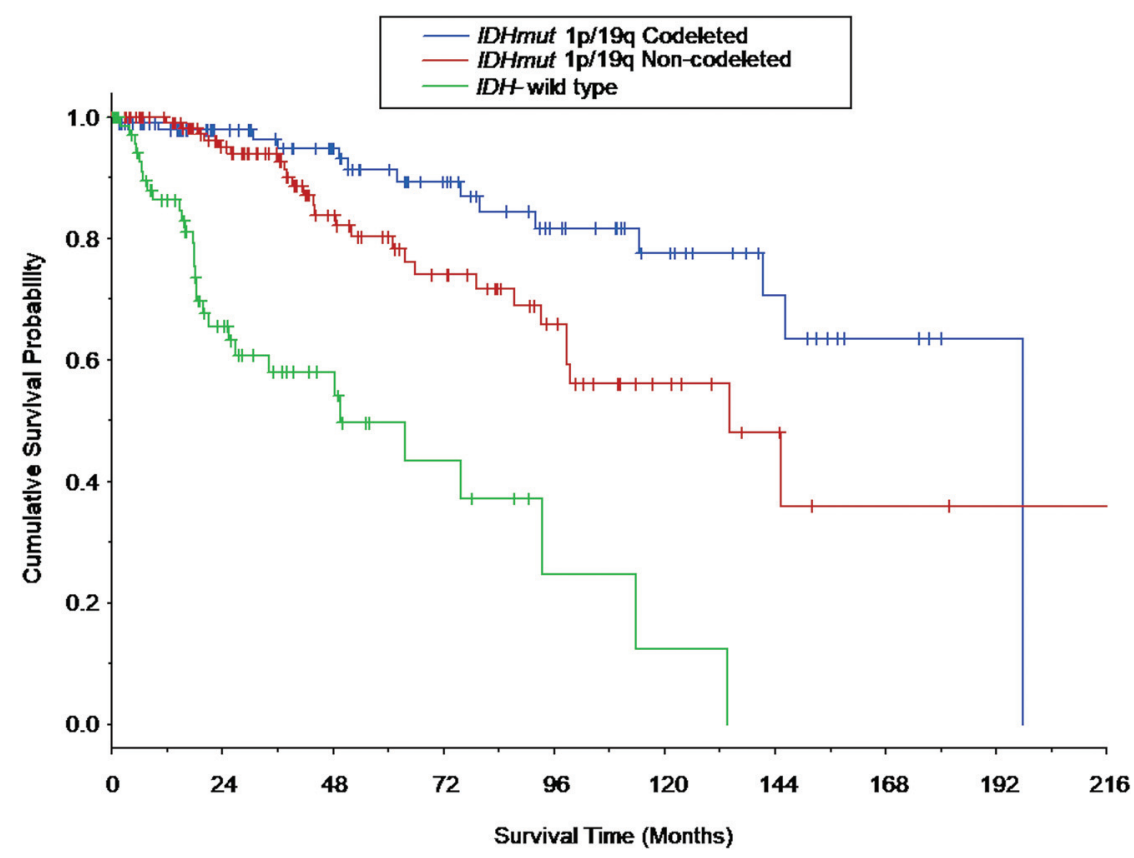

FIG 1. Kaplan-Meier survival curves based on the LGG molecular subtype. Vertical tick marks identify right-censored survival times. The survival curves differed among all 3 LGG molecular subtypes (IDH-mutant 1p/19q-codeleted molecular subtype versus IDH-mutant 1p/19q-noncodeleted molecular subtype: $P=.021$; IDH-mutant $1 \mathrm{p} / 19 \mathrm{q}$-codeleted molecular subtype versus IDH wild-type molecular subtype: $P<.001$; and $I D H$-mutant $1 \mathrm{p} / 19 \mathrm{q}$-noncodeleted molecular subtype versus IDH-wild type molecular subtype: $P<.001$ ). Survival curves for patients who composed a subset of the current patient cohort are shown in Patel et $\mathrm{al}^{46}{ }^{46}$ in 2019.
19 p13 FISH Probe Kit; Abbott Laboratories, Abbott Park, IL). Histopathologic and molecular data for the TCIA cohort were retrieved from supplemental material in Ceccarelli et al. ${ }^{25}$

\section{Statistical Analysis}

Data Summarization. Categoric variables are summarized by frequencies and percentages, and continuous scaled data are summarized by the median, the interquartile range (IQR), and the range of the empiric distribution.

Survival Analyses. Cox proportional hazards regression was used to examine the prognostic utility of the preoperative MR imaging metrics to predict survival within the currently defined molecular categories of LGGs. The survival analyses were conducted per molecular category (ie, IDHmut-Codel, IDHmutNonCodel, and IDHwt) and per MR imaging metrics. For each MR imaging metric, a multivariate Cox proportional hazards regression analysis was conducted in which the MR imaging metric of interest served as the primary predictor variable and patient age, patient sex, WHO grade, and surgical resection status served as concomitant adjustment variables. The follow-up times of survivors were treated as right-censored survival times in the Cox model. With

Table 3: Associations between preoperative MR imaging metrics and overall survival per LGG molecular subtype, after adjustment for patient age, sex, tumor grade, and surgical resection status ${ }^{\mathrm{a}}$

\begin{tabular}{|c|c|c|c|}
\hline Relative Comparison (Non-Reference:Reference) ${ }^{\mathrm{b}}$ & IDHmut-Codel & IDHmut-Noncodel & IDHwt \\
\hline Tumor size (3rd to 1st quartile) & $2.85(1.06-7.70)(P=.039)$ & $2.90(1.54-5.48)(P<.001)$ & $3.82(1.94-7.75)(P<.001)$ \\
\hline Location (central:peripheral) & $0.59(0.16-2.18)(P=.430)$ & $0.50(0.22-1.17)(P=.101)$ & $0.78(0.36-1.70)(P=.534)$ \\
\hline No. of involved lobes $(X+1: X)$ & $1.37(0.84-2.23)(P=.211)$ & $1.25(0.99-1.58)(P=.065)$ & $1.70(1.28-2.27)(P=.001)$ \\
\hline Corpus callosum extension (yes:no) & $2.60(0.69-9.84)(P=.160)$ & $1.42(0.59-3.41)(P=.433)$ & $1.89(0.78-4.58)(P=.160)$ \\
\hline Hydrocephalus (yes:no) & $2.57(0.42-15.81)(P=.308)$ & $0.63(0.19-2.11)(P=.457)$ & $4.43(1.12-17.54)(P=.034)$ \\
\hline Midline shift (3rd to 1st quartile) & $1.32(0.77-2.26)(P=.320)$ & $1.14(0.54-2.41)(P=.730)$ & $1.16(1.03-1.30)(P=.013)$ \\
\hline Eloquent cortex (yes:no) & $1.00(0.29-3.43)(P=.740)$ & $1.91(0.77-4.78)(P=.165)$ & $2.01(0.90-4.49)(P=.087)$ \\
\hline Ependymal extension (yes:no) & $6.34(1.07-37.59)(P=.042)$ & $1.72(0.64-4.64)(P=.289)$ & $1.51(0.52-4.35)(P=.447)$ \\
\hline Margins (global test $P$ value) ${ }^{c}$ & $(P=.775)$ & $(P=.190)$ & $(P=.031)$ \\
\hline $33 \%-66 \%:<33 \%{ }^{\mathrm{d}}$ & $1.57(0.42-5.97)(P=.505)$ & $0.51(0.19-1.33)(P=.166)$ & $0.39(0.17-0.90)(P=.027)$ \\
\hline$>66 \%:<33 \%^{\mathrm{d}}$ & $0.98(0.09-10.85)(P=.985)$ & $0.39(0.13-1.19)(P=.098)$ & $0.24(0.06-1.05)(P=.057)$ \\
\hline$>66 \%: 33 \%-66 \%{ }^{d}$ & $0.62(0.06-6.40)(P=.688)$ & $0.77(0.24-2.38)(P=.646)$ & $0.63(0.14-2.87)(P=.554)$ \\
\hline Contrast enhancement (yes:no) & $3.11(0.71-13.68)(P=.132)$ & $1.67(0.62-4.51)(P=.313)$ & $0.34(0.13-0.90)(P=.030)$ \\
\hline Necrosis (yes:no) & $1.84(0.32-10.78)(P=.498)$ & $0.28(0.03-2.42)(P=.247)$ & $1.93(0.82-4.58)(P=.134)$ \\
\hline
\end{tabular}

${ }^{a}$ Data are listed as adjusted hazard ratios ( $95 \%$ confidence interval) and corresponding $P$ values.

${ }^{b}$ Relative comparison (nonreference:reference) identifies the nonreference predictor variable value/level and the reference predictor variable value/level at which the adjusted instantaneous risk of death ratio (ie, hazard ratio) was evaluated. For example (3rd:1st quartile) represents a comparison of the adjusted instantaneous risk of death (ie, hazard) between 2 patients, 1 patient whose predictor variable value is at the 3 rd quartile of the predictor variable empiric distribution (nonreference) and 1 patient whose predictor variable value is at the 1st quartile of the predictor variable empiric distribution (reference). A relative comparison denoted as $(X+1: 1)$ represents a comparison of the adjusted instantaneous risk of death (ie, hazard) between 2 patients, 1 patient whose predictor variable value is $X+1$ units (nonreference) and 1 patient whose predictor variable value is $X$ units (reference). A relative comparison, denoted as (yes:no), represents a comparison of the adjusted instantaneous risk of death (ie, hazard) between 2 patients, 1 patient who has the factor of interest (nonreference $=$ yes) and 1 patient who does not have the factor of interest (reference $=$ no). Note, if the adjusted hazard ratio is $>1(<1)$, it indicates that the estimate for the instantaneous risk of death is greater (less) for the patient who has the nonreference predictor value/level.

"Global test $P$ value is the $P$ value for testing the null hypothesis that the instantaneous risk of death is same for all "margin" categories.

${ }^{d}$ Percentage of LGGs displaying sharp/circumscribed margin. 

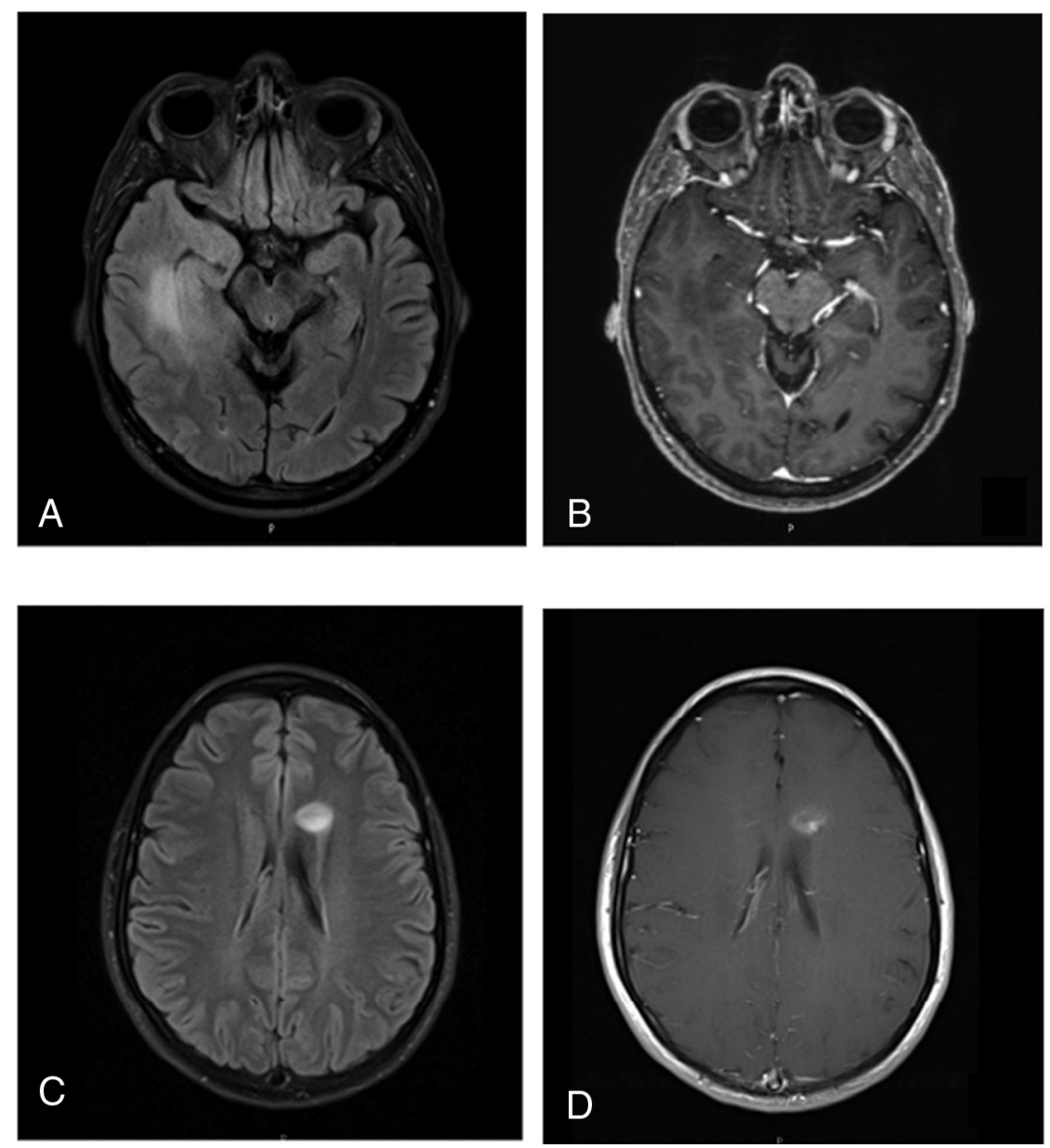

FIG 2. Representative cases of IDHwt LGGs. A 51-year-old man with an IDH wild-type diffuse astrocytoma. FLAIR $(A)$ shows a $6-\mathrm{cm}$ mass in the right temporal lobe with ill-defined margins. Contrast-enhanced TIWI (B) shows no contrast enhancement of the mass. This patient is deceased, with a survival time of 262 days. Further molecular testing in this case was positive for TERT promoter (-124 C > T) mutation. A 26-year-old woman with an IDH wild-type diffuse astrocytoma. FLAIR $(C)$ and contrast-enhanced TIWI $(D)$ show a $1.6-\mathrm{cm}$ left frontal lobe mass with fairly well-circumscribed margins and contrast enhancement. This patient was alive at last follow-up, with a survival time of 2757 days. Further molecular testing in this case was positive for the BRAF V600E mutation.

regard to hypothesis testing, the Wald $\chi^{2}$ test was used to test the null hypothesis that the instantaneous risk of death (ie, hazard) is not associated with the values/categories of the MR imaging metric of interest after adjustment for patient age, patient sex, WHO grade, and surgical resection status. A $P \leq .05$ decision rule was used as the null hypothesis rejection criterion for the tests of association.

Tumor Grade Analyses. MR imaging metrics versus WHO grade association were examined via multivariate logistic regression, per LGG molecular subtype. The dependent variable of the multivariate logistic regression model was an indicator variable $(\mathrm{Y})$ that distinguished WHO grade III tumors $(\mathrm{Y}=1)$ from WHO grade II tumors $(\mathrm{Y}=0)$, and the MR imaging metrics served as the predictor variables. Regarding hypothesis testing, the type III Wald $\chi^{2}$ test was used to test the null hypothesis that the adjusted odds for a tumor being WHO grade III are not associated with the MR imaging metric. A $P \leq .05$ decision rule was used as the null hypothesis rejection criterion.

\section{RESULTS}

The study population included 306 patients, with 154 women $(50.3 \%)$ and 152 men (49.7\%). Patient characteristics per LGG molecular subtype are shown in Table 1, and preoperative MR imaging metric empiric distribution summaries per LGG molecular subtype are shown in Table 2.

Survival curves for overall survival (OS) are shown in Fig 1 for the LGG molecular subtypes. OS differed among all the LGG molecular subtypes $(P<.022$ for all comparisons), with patients with IDHwt LGGs having the shortest median OS (49.5 months; 95\% CI lower bound, 25.5 months), followed by the patients with IDHmut-Noncodel (134.6 months; 95\% CI lower bound, 98.7 months), and patients with IDHmut-Codel (196.6 months, 95\% CI lower bound, 141.4 months).

The associations between preoperative MR imaging metrics and OS per LGG molecular subtype are expressed as hazard ratios (HRs) in Table 3, after adjustment for patient age, patient sex, tumor grade, and surgical resection status. For IDHwt LGGs, greater tumor size (HR, 3.82; 95\% CI, 1.94-7.75; $P<.001)$, greater number of involved lobes (HR, 1.70; 95\% CI, 1.28-2.27; $P<.001$ ), hydrocephalus (HR, 4.43; 95\% CI, 1.12-17.54; $P=.034)$, greater degree of midline shift (HR, 1.16; 95\% CI, $1.03-1.30 ; P=.013)$, and less circumscribed margins $(P=.031)$ were associated with shorter OS, whereas the presence of contrast enhancement (HR, 0.34; 95\% CI, $0.13-0.90 ; P=.030$ ) was associated with longer OS (Fig 2). For IDHmut-Noncodel LGGs, greater tumor size (HR, 2.90; 95\% CI, 1.54-5.48; $P=.001$ ) was associated with shorter OS. For IDHmut-Codel LGGs, greater tumor size (HR, 2.85; 95\% CI, $1.06-7.70 ; P=.039$ ) and ependymal extension (HR, 6.34; $95 \% \mathrm{CI}$, $1.07-37.59 ; P=.042)$ were associated with shorter OS.

A secondary analysis determined unique associations between MR imaging metrics and tumor grade (II or III) per LGG molecular subtype (Table 4). Contrast enhancement was associated with grade III for both IDHwt LGGs (OR, 16.75; 95\% CI, 3.47-80.86; $P<.001)$ and IDHmut-Noncodel LGGs (OR, 6.08; 95\% CI, 2.12-17.41; $P=.001$ ), but not for IDHmut-Codel LGGs. For IDHwt LGGs, ependymal extension was associated with grade II (OR, 0.09; 95\% CI, $0.01-$ $0.59 ; P=.012$ ), and for IDHmut-Noncodel LGGs, central 
Table 4: Associations between preoperative MR imaging metrics and WHO grade III per molecular subtype ${ }^{\mathrm{a}}$

\begin{tabular}{|c|c|c|c|}
\hline Relative Comparison (Non-Reference:Reference) ${ }^{b}$ & IDHmut-Codel & IDHmut-Noncodel & IDHwt \\
\hline Tumor size (3rd to lst quartile) & $0.97(0.18-5.26)(P=.975)$ & $1.24(0.34-4.57)(P=.742)$ & $1.60(0.31-8.17)(P=.573)$ \\
\hline Location (central:peripheral) & $1.55(0.22-10.49)(P=.654)$ & $0.21(0.05-0.80)(P=.035)$ & $0.42(0.10-1.84)(P=.252)$ \\
\hline No. of involved lobes $(X+1: X)$ & $1.45(0.64-3.30)(P=.370)$ & $1.12(0.65-1.94)(P=.673)$ & $0.96(0.48-1.92)(P=.906)$ \\
\hline Corpus callosum extension (yes:no) & $1.88(0.39-9.05)(P=.430)$ & $2.51(0.72-8.76)(P=.149)$ & $1.46(0.25-8.35)(P=.672)$ \\
\hline Hydrocephalus (yes:no) & $8.55(0.33-223.32)(P=.197)$ & $2.07(0.50-8.54)(P=.313)$ & $0.26(0.01-5.09)(P=.372)$ \\
\hline Midline shift (3rd to 1st quartile) & $0.88(0.37-2.10)(P=.774)$ & $0.55(0.16-1.85)(P=.331)$ & $1.31(0.91-1.89)(P=.148)$ \\
\hline Eloquent cortex (yes:no) & $1.71(0.49-5.99)(P=.402)$ & $1.87(0.69-5.09)(P=.220)$ & $0.35(0.08-1.54)(P=.165)$ \\
\hline Ependymal extension (yes:no) & $1.68(0.28-10.07)(P=.570)$ & $0.65(0.15-2.86)(P=.571)$ & $0.09(0.01-0.59)(P=.012)$ \\
\hline Margins (global test) & $P=.970$ & $P=.360$ & $P=.221$ \\
\hline $33 \%-66 \%:<33 \%{ }^{d}$ & $1.20(0.28-5.23)(P=.804)$ & $1.47(0.37-5.79)(P=.583)$ & $1.62(0.24-10.84)(P=.619)$ \\
\hline$>66 \%:<33 \%^{\mathrm{d}}$ & $1.16(0.15-9.17)(P=.888)$ & $0.64(0.12-3.56)(P=.614)$ & $0.27(0.02-3.35)(P=.305)$ \\
\hline$>66 \%: 33 \%-66 \%^{d}$ & $0.96(0.17-5.56)(P=.967)$ & $0.44(0.13-1.42)(P=.170)$ & $0.16(0.02-1.28)(P=.084)$ \\
\hline Contrast enhancement (yes:no) & $1.73(0.47-6.43)(P=.411)$ & $6.08(2.12-17.41)(P=.001)$ & $16.75(3.47-80.86)(P<.001)$ \\
\hline Necrosis (yes:no) & $9.68(0.83-112.49)(P=.070)$ & $0.61(0.08-4.67)(P=.632)$ & $0.67(0.13-3.36)(P=.630)$ \\
\hline
\end{tabular}

${ }^{a}$ Note that for the multivariate logistic regression analysis, WHO grade III tumors were assigned the value 1 and WHO grade II tumors were assigned the value 0 . Data are listed as adjusted odds ratios, (95\% confidence interval) and corresponding $P$ value determined by a Wald type III $\chi^{2}$ test.

${ }^{b}$ Relative comparison (nonreference:reference) identifies the nonreference predictor variable value/level and the reference predictor variable value/level at which the adjusted odds ratio was evaluated. For example (3rd:1st Quartile) represents a comparison of the adjusted odds for tumor grade III between 2 patients, 1 patient whose predictor variable value is at the 3rd quartile of the predictor variable empiric distribution (nonreference) and 1 patient whose predictor variable value is at the 1st quartile of the predictor variable empiric distribution (reference). A relative comparison denoted as ( $\mathrm{X}+1: 1)$ represents a comparison of the adjusted odds for tumor grade III between 2 patients, 1 patient whose predictor variable value equals $X+1$ (nonreference) and 1 patient whose predictor variable value equals $X$ (reference). A relative comparison denoted as (yes:no) represents a comparison of the adjusted odds for a tumor grade III between 2 patients, 1 patient who has the factor of interest (nonreference $=$ yes) and 1 patient who does not have the factor of interest (reference $=$ no). Note, if the adjusted odds ratio is $>1(<1)$, it indicates that the estimate for the adjusted odds for a tumor grade III is greater (less) for the patient who has the nonreference predictor value/level.

"Global test $P$ value is the $P$ value for testing the null hypothesis that the adjusted odds ratio is same for all "margin" categories.

${ }^{d}$ Percentage of LGG displaying sharp/circumscribed margin.

location was associated with grade II (OR, 0.21; 95\% CI, $0.05-0.80 ; P=.035)$.

\section{DISCUSSION}

Despite the markedly improved prognostic relevance of the 2016 WHO classification of LGGs, clinical outcome variability persists for LGGs within existing molecular subtypes. ${ }^{26-29}$ For each LGG molecular subtype, we separately explored the association between preoperative MR imaging metrics and patient OS after adjustment for WHO grade, surgical resection status, patient age, and patient sex. We further explored the relationship between MR imaging metrics and tumor grade separately for each LGG subtype. Among our results, we found that preoperative MR imaging features have more prognostic value for IDHwt LGGs than for IDH-mutant LGGs. Tumor size is significantly associated with OS in all LGG molecular subtypes, and MR imaging associations with tumor grade vary among LGG molecular subtypes.

Our finding that preoperative MR imaging has the greatest prognostic value for the IDHwt subtype is potentially explained by the increasingly well-recognized biologic and clinical heterogeneity of IDHwt LGGs, in particular based on molecular alterations such as telomerase reverse transcriptase (TERT) promoter mutation (Fig 2A,-B), epidermal growth factor receptor (EGFR) gene amplification, and chromosome $7 / 10$ alterations. $^{12,26,27,30}$ These molecular alterations have not yet been formally incorporated into the WHO classification scheme, and it is conceivable that some of our neuroimaging metrics correlated with these prognostically significant molecular alterations among our IDHwt cohort. We further speculate that our apparently counterintuitive finding of a positive correlation between contrast enhancement and survival time for the IDHwt cohort could be explained by the potential inclusion of B-raf proto-oncogene
(BRAF)-mutant IDHwt gliomas in our cohort (Fig 2C, -D). $B R A F$-mutant diffuse gliomas commonly demonstrate contrast enhancement and are associated with favorable clinical outcomes. $^{31-33}$

On the other hand, we found relatively few significant associations with overall survival for the $I D H$-mutant LGG subtypes. These results might reflect that the IDH-mutant LGG subtypes represent more uniform genomic entities compared with the IDHwt subtype. Nonetheless, for both IDHmut-Noncodel and IDHmut-Codel subtypes, larger tumor size was associated with worse OS. Moreover, we found that ependymal extension is associated with worse OS for the IDHmut-Codel subtype. It is known that subventricular zone extension by glioblastoma portends a worse prognosis, possibly due to recruitment of migratory progenitor cells in this location. ${ }^{34}$ It is unclear whether a similar process contributes to a more aggressive clinical course among the IDHmut-Codel LGGs, and further investigation into the impact of ependymal extension in the IDHmut-Codel subtype could build on our results.

To our knowledge, the literature investigating prognostically relevant imaging metrics in molecularly defined LGG subtypes is sparse. Perhaps the most applicable study is by $\mathrm{Wu}$ et $\mathrm{al},{ }^{35}$ in 2019, who found that tumor size and elevated relative cerebral blood volume on preoperative imaging correlate with a more aggressive subtype of IDHmut-Noncodel gliomas. While our results support these findings in regard to tumor size, we observed a similar relationship for IDHmut-Codel and IDHwt subgroups as well. Small sample size and lack of adjustment for WHO grade by $\mathrm{Wu}$ et al are limitations that may have contributed to differences observed between our studies. Suchorska et $\mathrm{al}^{36}$ in 2019, found that contrast enhancement is a viable prognostic metric for $I D H$-mutant subgroups, a finding that was not reproduced in our study. Finally, the apparent diffusion 
coefficient has been reported as a potential prognostic marker for IDHwt LGGs. ${ }^{37}$

Multiple prior studies have investigated imaging findings that correlate with WHO grade. ${ }^{38-42}$ However, to the best of our knowledge, we are the first to report the relationship between neuroimaging metrics and WHO grade separately for each LGG molecular subtype. Our results indicate that contrast enhancement predicts WHO grade III for the IDHmutNoncodel and IDHwt LGGs, but not for IDHmut-Codel LGGs. A previous study of oligodendrogliomas by White et $\mathrm{al},{ }^{43}$ in 2005 , also reported no association between contrast enhancement and WHO grade; however, 1p/19q testing was not undertaken in their study, and their cohort was small $(n=24)$. Our results strengthen this conclusion and may serve to modify the well-established dictum that contrast enhancement correlates with tumor grade in adult diffuse gliomas. $^{44,45}$

While efforts to identify preoperative neuroimaging predictors of LGG molecular status are valuable, the clinical utility is frequently limited because nearly all such cases undergo biopsy or resection for a definitive pathologic diagnosis. Our investigation aimed to uncover simple neuroimaging metrics that could relay prognostic information for LGGs beyond what can be inferred from their molecular and histologic characterization. An added strength of our study design was the adjustment of our analysis for both tumor grade and surgical resection status. The extent of surgical resection in particular is well-recognized for its impact on the overall survival of a patient with LGG. ${ }^{46,47}$

Nonetheless, our study has limitations. This is a retrospective study and thus inherently limited by design in its ability to infer relationships beyond association. Further prospective multi-institutional investigations would be necessary to confirm our results. Another potential limitation is the binary method of analysis for several metrics (eg, yes or no contrast enhancement). While this method was chosen to reduce ambiguity in the data acquisition and maintain a simple set of imaging metrics, a more graded approach to measurement may allow a more nuanced and potentially revealing analysis. Similarly, we measured "tumor size" as a single long-axis diameter, and volumetric measurements based on 3D MR imaging acquisitions would be preferable. Furthermore, we investigated a limited set of MR imaging pulse sequences (precontrast T1WI, contrast-enhanced T1WI, and either T2WI or FLAIR), and additional study into the prognostic value of more advanced techniques (eg, perfusion-weighted imaging, diffusion tensor imaging, susceptibility-weighted imaging) might be fruitful. We did not adjust our analysis for nonsurgical treatment (eg, chemotherapy and radiation therapy), which presumably varied across our cohort. Finally, future investigations evaluating links between MR imaging metrics and molecular alterations pertinent to IDHwt LGGs, such as BRAF or TERT mutations, would be highly valuable.

\section{CONCLUSIONS}

Preoperative MR imaging metrics offer prognostic information for patients with LGG within molecularly defined classes. Multiple neuroimaging features had unique prognostic significance for the IDHwt subtype, including hydrocephalus, midline shift, margin features, and contrast enhancement, likely reflecting the known biologic and clinical heterogeneity of IDHwt LGGs. Contrast enhancement was associated with WHO grade III among IDHwt and IDHmut-Noncodel LGGs, but not IDHmutCodel LGGs.

Disclosures: Laila M. Poisson-UNRELATED: Grants/Grants Pending: National Cancer Institute, Comments: R01CA222146.* Maria-Beatriz Lopes-UNRELATED: Grants/Grants Pending: Focus Ultrasound Foundation*; Royalties: Elsevier, Comments: textbook chapter. Rajan Jain-UNRELATED: Consultancy: Cancer Panels; Royalties: Thieme. Camilo E. Fadul_UNRELATED: Grants/Grants Pending: Novartis.* David Schiff_UNRELATED: Board Membership: Orbus Therapeutics, Comments: Data and Safety Monitoring Committee Chair for clinical trial; Royalties: UpToDate. Sohil H. Patel—RELATED: Grant: Radiological Society of North America Scholar Grant, Comments: RSCH1819.* *Money paid to the institution.

\section{REFERENCES}

1. Brat DJ, Verhaak RG, Aldape KD, et al; Cancer Genome Atlas Research Network. Comprehensive, integrative genomic analysis of diffuse lower-grade gliomas. $N$ Engl J Med 2015;372:2481-98 CrossRef Medline

2. Eckel-Passow JE, Lachance DH, Molinaro AM, et al. Glioma groups based on 1p/19q, IDH, and TERT promoter mutations in tumors. N Engl J Med 2015;372:2499-2508 CrossRef Medline

3. Louis DN, Perry A, Reifenberger G, et al. The 2016 World Health Organization classification of tumors of the central nervous system: a summary. Acta Neuropathol 2016;131:803-20 CrossRef Medline

4. Yan H, Parsons DW, Jin G, et al. IDH1 and IDH2 mutations in gliomas. N Engl J Med 2009;360:765-73 CrossRef Medline

5. Chamberlain MC, Born D. Prognostic significance of relative $1 \mathrm{p} /$ $19 q$ codeletion in oligodendroglial tumors. $J$ Neurooncol 2015;125:249-51 CrossRef Medline

6. Aldape K, Burger PC, Perry A. Clincopathologic aspects of 1p/19q loss and the diagnosis of oligodendroglioma. Arch Pathol Lab Med 2007;131:242-51 Medline

7. Liu X, Gerges N, Korshunov A, et al. Frequent ATRX mutations and loss of expression in adult diffuse astrocytic tumors carrying IDH1/IDH2 and TP53 mutations. Acta Neuropathol 2012;124:61525 CrossRef Medline

8. Kannan K, Inagaki A, Silber J, et al. Whole-exome sequencing identifies ATRX mutation as a key molecular determinant in lowergrade glioma. Oncotarget 2012;3:1194-1203 CrossRef Medline

9. Reuss DE, Kratz A, Sahm F, et al. Adult IDH wild type astrocytomas biologically and clinically resolve into other tumor entities. Acta Neuropathol 2015;130:407-17 CrossRef Medline

10. Gleize V, Alentorn A, Connen de Kérillis L, et al; POLA network. CIC inactivating mutations identify aggressive subset of $1 p 19 q$ codeleted gliomas. Ann Neurol 2015;78:355-74 CrossRef Medline

11. Reis GF, Pekmezci M, Hansen HM, et al. CDKN2A loss is associated with shortened overall survival in lower-grade (World Health Organization grades II-III) astrocytomas. J Neuropathol Exp Neurol 2015;74:442-42 CrossRef Medline

12. Aibaidula A, Chan AK, Shi Z, et al. Adult IDH wild-type lowergrade gliomas should be further stratified. Neuro Oncol 2017;19:1327-37 CrossRef Medline

13. Park YW, Han K, Ahn SS, et al. Prediction of IDH1-mutation and $1 \mathrm{p} / 19 \mathrm{q}$-codeletion status using preoperative MR imaging phenotypes in lower grade gliomas. AJNR Am J Neuroradiol 2018;39:3742 CrossRef Medline

14. Lasocki A, Gaillard F, Gorelik A, et al. MRI features can predict 1p/ $19 q$ status in intracranial gliomas. AJNR Am J Neuroradiol 2018;39:687-92 CrossRef Medline

15. Smits M, van den Bent MJ. Imaging correlates of adult glioma genotypes. Radiology 2017;284:316-31 CrossRef Medline 
16. Jenkinson MD, Du Plessis DG, Smith TS, et al. Histological growth patterns and genotype in oligodendroglial tumours: correlation with MRI features. Brain 2006;129:1884-91 CrossRef Medline

17. Megyesi JF, Kachur E, Lee DH, et al. Imaging correlates of molecular signatures in oligodendrogliomas. Clin Cancer Res 2004;10:4303-06 CrossRef Medline

18. Patel SH, Poisson LM, Brat DJ, et al. T2-FLAIR mismatch, an imaging biomarker for IDH and $1 \mathrm{p} / 19 \mathrm{q}$ status in lower-grade gliomas: a TCGA/TCIA project. Clin Cancer Res 2017;23:6078-85 CrossRef Medline

19. Batchala PP, Muttikkal TJ, Donahue JH, et al. Neuroimaging-based classification algorithm for predicting $1 \mathrm{p} / 19 \mathrm{q}$-codeletion status in IDH-mutant lower grade gliomas. AJNR Am J Neuroradiol 2019;40:426-32 CrossRef Medline

20. Aliotta E, Nourzadeh H, Batchala P, et al. Molecular subtype classification in lower-grade glioma with accelerated DTI. AJNR Am J Neuroradiol 2019;40:1458-63 CrossRef Medline

21. Radiology Data from The Cancer Genome Atlas Low Grade Glioma [TCGA-LGG] collection. The Cancer Imaging Archive. https://www. cancerimagingarchive.net. Accessed April 11, 2019

22. Chang EF, Smith JS, Chang SM, et al. Preoperative prognostic classification system for hemispheric low-grade gliomas in adults. $J$ Neurosurg 2008;109:817-24 CrossRef Medline

23. Capper D, Weissert S, Balss J, et al. Characterization of $\mathbf{R} 132 \mathrm{H}$ mutation-specific IDH1 antibody binding in brain tumors. Brain Pathol 2010;20:245-54 CrossRef Medline

24. Capper D, Zentgraf H, Balss J, et al. Monoclonal antibody specific for IDH1 R132H mutation. Acta Neuropathol 2009;118:599-601 CrossRef Medline

25. Ceccarelli M, Barthel FP, Malta TM, et al; TCGA Research Network. Molecular profiling reveals biologically discrete subsets and pathways of progression in diffuse glioma. Cell 2016;164:550-63 CrossRef Medline

26. Di Carlo DT, Duffau H, Cagnazzo F, et al. IDH wild-type WHO grade II diffuse low-grade gliomas: a heterogeneous family with different outcomes-systematic review and meta-analysis. Neurosurg Rev 2018 Jun 26. [Epub ahead of print] CrossRef Medline

27. Poulen G, Goze C, Rigau V, et al. Huge heterogeneity in survival in a subset of adult patients with resected, wild-type isocitrate dehydrogenase status, WHO grade II astrocytomas. J Neurosurg 2018 Apr 20. [Epub ahead of print] CrossRef Medline

28. Wijnenga M, French PJ, Dubbink HJ, et al. Prognostic relevance of mutations and copy number alterations assessed with targeted next generation sequencing in IDH mutant grade II glioma. $J$ Neurooncol 2018;139:349-57 CrossRef Medline

29. Wijnenga MM, Dubbink HJ, French PJ, et al. Molecular and clinical heterogeneity of adult diffuse low-grade IDH wild-type gliomas: assessment of TERT promoter mutation and chromosome 7 and 10 copy number status allows superior prognostic stratification. Acta Neuropathol 2017;134:957-59 CrossRef Medline

30. Brat DJ, Aldape K, Colman H, et al. cIMPACT-NOW update 3: recommended diagnostic criteria for "Diffuse astrocytic glioma, IDH-wildtype, with molecular features of glioblastoma, WHO grade IV." Acta Neuropathol 2018;136:805-10 CrossRef Medline
31. Chi AS, Batchelor T, Yang D, et al. BRAF V600E mutation identifies a subset of low-grade diffusely infiltrating gliomas in adults. J Clin Oncol 2013;31:e233-36 CrossRef Medline

32. Chan AY, Zhang RQ, Aibaidula A, et al. BRAF mutation marks out specific subgroups of glioma. Glioma 2018;1:168 CrossRef

33. Aibaidula A, Chan AK, Shi Z, et al. Adult IDH wild-type lowergrade gliomas should be further stratified. Neuro-Oncology 2017;19:1327-37 CrossRef Medline

34. Jafri NF, Clarke JL, Weinberg V, et al. Relationship of glioblastoma multiforme to the subventricular zone is associated with survival. Neuro-Oncology 2013;15:91-96 CrossRef Medline

35. Wu CC, Jain R, Neto L, et al. MR imaging phenotype correlates with extent of genome-wide copy number abundance in IDH mutant gliomas. Neuroradiology 2019;61:1023-31 CrossRef Medline

36. Suchorska B, Schüller U, Biczok A, et al. Contrast enhancement is a prognostic factor in IDH1/2 mutant, but not in wild-type WHO grade II/III glioma as confirmed by machine learning. Eur J Cancer 2019;107:15-27 CrossRef Medline

37. Wu C, Jain R, Radmanesh A, et al. Predicting genotype and survival in glioma using standard clinical MR imaging apparent diffusion coefficient images: a pilot study from the Cancer Genome Atlas. AJNR Am J Neuroradiol 2018;39:1814-20 CrossRef Medline

38. Hakyemez B, Erdogan C, Ercan I, et al. High-grade and low-grade gliomas: differentiation by using perfusion MR imaging. Clin Radiol 2005;60:493-502 CrossRef Medline

39. Komatsu K, Wanibuchi M, Mikami T, et al. Arterial spin labeling method as a supplemental predictor to distinguish between high- and low-grade gliomas. World Neurosurg 2018;114:e495500 CrossRef Medline

40. Togao O, Hiwatashi A, Yamashita K, et al. Differentiation of highgrade and low-grade diffuse gliomas by intravoxel incoherent motion MR imaging. Neuro-Oncology 2016;18:132-41 CrossRef Medline

41. Leu K, Ott GA, Lai A, et al. Perfusion and diffusion MRI signatures in histologic and genetic subtypes of WHO grade II-III diffuse gliomas. J Neurooncol 2017;134:177-88 CrossRef Medline

42. Delgado AF, Delgado AF. Discrimination between glioma grades II and III using dynamic susceptibility perfusion MRI: a meta-analysis. AJNR Am J Neuroradiol 2017;38:1348-55 CrossRef Medline

43. White M, Zhang Y, Kirby P, et al. Can tumor contrast enhancement be used as a criterion for differentiating tumor grades of oligodendrogliomas? AJNR Am J Neuroradiol 2005;26:784-90

44. Smirniotopoulos JG, Murphy FM, Rushing EJ, et al. Patterns of contrast enhancement in the brain and meninges. Radiographics 2007;27:525-51 CrossRef Medline

45. Young GS. Advanced MRI of adult brain tumors. Neurol Clin 2007;25:947-73 CrossRef Medline

46. Patel SH, Bansal AG, Young EB, et al. Extent of surgical resection in lower-grade glioma: differential impact based on molecular subtype. AJNR Am J Neuroradiol 2019;40:1149-55 CrossRef Medline

47. Wijnenga MM, French PJ, Dubbink HJ, et al. The impact of surgery in molecularly defined low-grade glioma: an integrated clinical, radiological, and molecular analysis. Neuro Oncol 2018;20:103-12 CrossRef Medline 